\title{
Overlay IoT Network Architecture Using Packet Aggre- gation and Disaggregation
}

\author{
Arata Koike *, Takumi Ohba , \\ Ryota Ishibashi *
}

\begin{abstract}
We anticipate digital social innovation for improving our life and society. Internet of Things (IoT) is now considered as a key driving force for achieving such next generation social systems. Several approaches have been proposed to achieve effective IoT communications, where each device has very limited communication capabilities. One such approach is the introduction of short packets for a lightweight communication protocol by which we can form a local area sensor network dedicated to IoT devices and can achieve effective packet processing, reducing power consumption, and minimizing bandwidth occupancy. The short packet can solve prob-lems regarding local networks, but creates another problem for the wide area Internet, where various devices and local networks are converged. We propose an aggregation scheme that makes it possible to reduce power consumption and packet processing loads caused by a huge amount of short packets. We especially focus on the wide area Internet with our proposed scheme to support IoT traffic more efficiently by showing our CoAP-based implementation.
\end{abstract}

Keywords: aggregation, CoAP, Internet of Things, overlay network.

\section{Introduction}

Internet of Things (IoT) is attracting a great deal of attention. Although there are many different interpretations of IoT from various aspects, we here categorize it as a communication system for devices such as sensors. Communications for IoT are not directly adhered to communications among humans. The Internet, our conventional communication system, involves devices such as mobile phones and personal computers, which humans operate. When the Internet embraces IoT devices, we have to deal with various types of traffic inside the Internet, i.e., traffic not only for human communications, but also for many machine-type communications such as data from thermal and radiation sensors, smart meters, and control messages for actuators or robots. We anticipate that non-human communication will lead to the evolution of the Internet toward be-coming a more advanced communication system for our next generation social systems.

* Nippon Telegraph and Telephone Corp., Tokyo, Japan 
Unlike personal computers for current web browsing, IoT devices such as sensors, smart meters, and actuators are communication equipment with many resource constraints. For example, many of these devices use low capacity batteries without replacing them for several years; henceforth, highly efficient power saving capability is required. They also only have a limited amount of memory, which results in a lightweight software implementation footprint for communication. This means that we are unable to use widely adopted technologies for the Web, such as Hyper-text Transfer Protocol (HTTP) [1], as they are. Since HTTP protocol creates rather large length of messages, it consumes a large amount of power and memory space.

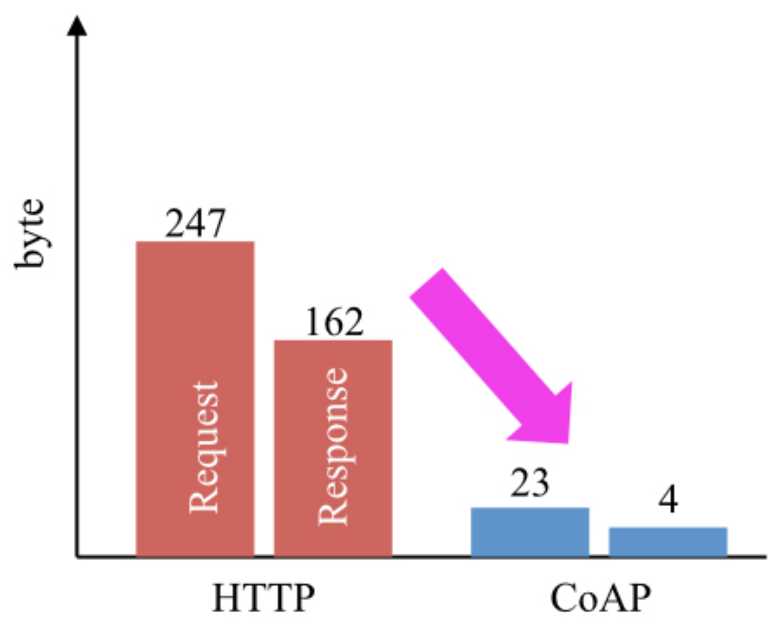

Figure 1(a): Message size (excluding layer 3 and 4 overhead) in byte

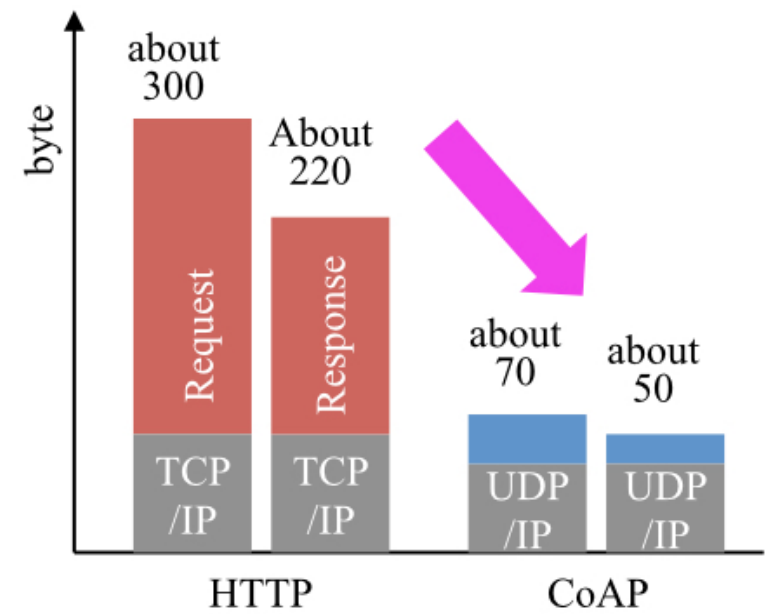

Figure 1(b): Message size (including layer 3 and 4 overhead) in byte 
There is a strong need for new technologies that enable communications for such resource-constrained equipment. The Constrained Application Protocol (CoAP) is one such lightweight communication technology [2] (Figure 1(a) and Figure 1(b)).

Reducing the message size as much as possible is one of the major design objectives with the CoAP. By reducing the message size, we can reduce power consumption during communication. The smaller the message size is the shorter communication duration we need. This makes it possible to extend the battery life of resource-constrained devices, such as sensors, smart meters, and actuators.

We next consider the case in which there are so many of these constrained devices that communicate each other or are connected to servers traveling through the wide-area Internet. In this case, there are quite a few short packets transmitted in wide area networks. In general, the Internet is established by the interconnection of networks by routers. We usually understand router performance in bits per second (bps) throughput. There, however, is another limit of the number of processing packets per second (pps). In other words, even if the throughput, bps, does not reach the capacity of a router, performance degradation of the router may occur when its pps increases. The increase in packet processing load results in an increase in power consumption, larger packet processing delays, and packet losses. Also, as each short packet has its own header, the overhead increases compared to a longer packet. This results in an inefficient bandwidth usage.

In this paper, we focus on packet aggregation techniques that make it possible to reduce power consumption and processing load at a router caused by a huge amount of short packets. Many packet aggregation techniques for IoT have been studied for local area networks to achieve efficiency. Our approach is rather targeting wide area networks. We study aggregation techniques from the viewpoint of how can we support IoT traffic in wide area network including the fixed and mobile wide area Internet or data center area networks. Note that the earlier concept version of this thought was presented in [3].

This paper is organized as follows. In Section II, we summarize existing aggregation techniques for several areas of networking researches including IoT and point out issues when we apply them to the wide area Internet in the context of IoT. We identify requirements for applying aggregation techniques for the wide area Internet in Section III. We discuss our proposed aggregation scheme, its architecture and, procedures for applying it to the wide area Internet in Section IV. We also show the format and sequence of our prototype implementation using CoAP. In Section V, we summarize our paper and discuss remaining issues and future prospect from the viewpoint of the construction of overlaying network.

\section{Packet Aggregations for Various Area on Networking Re- search}

There have mainly been three areas where packet aggregation schemes are applied: voice encoding, high-speed wireless network, and IoT area network. Roughly speaking, we can categorize these schemes as aiming for the improvements of bandwidth efficiencies. We first briefly review these schemes to clarify their objectives.

\subsection{Voice over Packet-based Network}


We can see packet aggregation method in the area of encoding voice for transmitting over packet-based networks [4][5][6]. When we transmit real-time voice or sound over packet-based network, it is inefficient to carry an element of sound per packet. For example, in Voice over IP (VoIP), we use RTP over UDP as its transport layer protocol [4]. Even if we pick up layer 3 and layer 4, UDP has 8 bytes and IP has 20 bytes headers respectively. On the other hand, when the sound is encoded by PCM [7], it adopts $8 \mathrm{kHz}$ sampling and 8bit quantization for digitizing the sound. This means it generates 1 byte of data in every 125 microseconds. When we generate a packet every 125 microseconds, it means it transports one byte of data with 28 bytes of overhead even if we think about layer 3 and layer 4 . This is very inefficient. Therefore, we aggregate several elements of sound into a packet. If we aggregate 160 sound elements, that means we only need to send a packet per 20 milliseconds. This aggregation highly reduces the overhead and achieves very efficient transmission.

When making this aggregation, we can easily recognize that we add additional delays to the sound element except the last one. If we consider end-to-end communication, we have to minimize the maximum delay to avoid deteriorating real-time property. Therefore, our requirements are reducing the overhead and minimizing the delay; these are two opposite directions of requirements and we have to balance them. For Asynchronous Transfer Mode (ATM) Voice and Telephony over ATM (VTOA), it introduces short cells. Short cells are aggregated into a regular 48-byte ATM cell. To minimize the delay, VTOA specification [5][6] allows short-cell based switching if intermediate ATM switch support a disaggregation and re-aggregation function.

\subsection{High-speed Wireless Network}

High-speed wireless networking is the second area for aggregation technique. The physical layer speed of IEEE 802.1n [8] is up to 600Mbps. Its specification, however, requires interframe gap and ack-based transmission for successive frames. Therefore, there is a waiting time for sending a successive frame. Henceforth, in order to increase the transmission efficiency, it is effective to reduce the number of frame and the specification adopts frame aggregation scheme.

\subsection{Communication protocols for IoT area network and aggregation techniques}

Sensors and smart meters are typical IoT devices that have limited battery capacity and processing capabilities. When we use these devices for IoT communication, we need to minimize packet size and number of transactions. Therefore, lightweight communication protocols such as 6LoWPAN-HC [9] and CoAP [2] are used. These protocols generate short packets compared to those of HTTP, which adopts a generally very long text message for its payload. On the other hand, the CoAP uses binary encoding for its payload for conveying control messages for devices with poor resources. CoAP dramatically reduces packet size. The CoAP has a fixed 4-byte header. Other fields are non-mandatory options so that the minimum packet size is only 4 bytes; this results in very small header overhead. The CoAP architecture consists of clients, servers, and proxies. A client sends requests for data retrieval, renewal, and removal. A server responds to a message from a client, and a proxy relays the CoAP messages. With 6LoWPAN-HC, the IP header is compressed in accordance with IEEE 802.15.4 [10]. This enables a lightweight protocol that adapts to the IEEE802.15.4 frame with 127 bytes. In order to reduce the number of transactions, aggregation techniques have been investigated [11][12]. Upon reception of multiple packets, those techniques combine individual packets and generate a single packet rather than transmit individual packets as they are. Merging multiple packets increases the effectiveness of 
packet processing, reduces power consumption, and reduces bandwidth usage for the rest part of a network.

There have been several studies on aggregation in such networks. Mlaih and Aly [13] studied a case in which multiple sensor devices form a multi-hop tree structure network toward a single destination sink node. When an intermediate sensor node receives successive packets from its leaf sensor nodes, it aggregates the multiple packets to a single packet and transmits it to its parent node. The parent node repeats the same process (Figure 2).

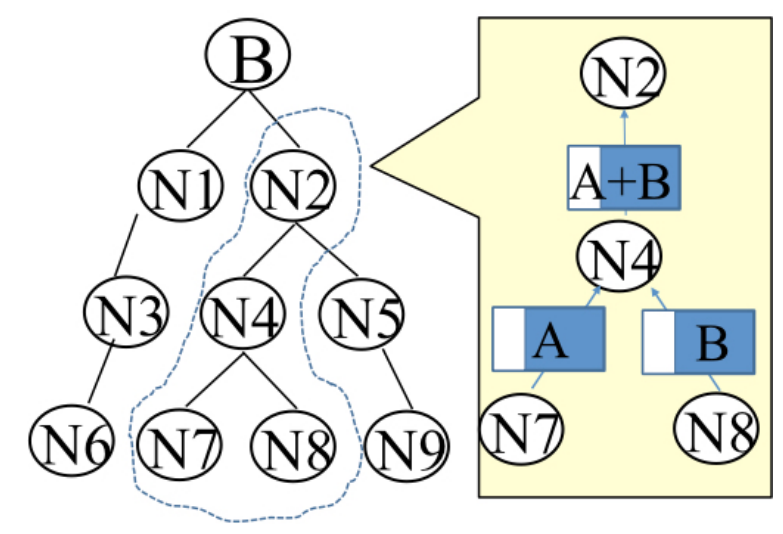

Figure 2: Packet aggregation in local area sensor network

When the intermediate sensor node receives data from multiple leaf sensor devices, it performs certain operations and transmits the result to the parent node. Example operations are summation or taking the average of multiple data. These operations create new data, and the node transfers the data to its next hop parent node. By repeating these operations from the beginning of the leaf to the root sink node, we can dramatically reduce the amount of data, reduce power consumption for communication, and efficiently use the restricted bandwidth.

\subsection{Issues of Aggregation in Wide Area Network and Datacenter Area Network}

As we looked at the typical application for aggregation, their primary objectives are improving the efficiency for transmission. For the first and second examples above, excessive delay is unacceptable so we also have an objective to minimize the delay for transmission. In this paper, we focus on packet aggregation in wide-area network for IoT. For IoT applications, although some IoT application such as robotics might have some delay requirements, we here assume that many of sensor applications do not have stringent delay requirements as they will have time stamp information inside their data. As for the transmission efficiency, amount of IoT traffic itself is very limited comparing to the other data traffic in wide area network. Since we usually have a large link capacity for core network, data transmission efficiency is not an issue for wide area network. Thus, in this paper, we focus on packet exchange efficiency for IoT traffic due to large number of small packets. This means maximizing link efficiency or minimizing delay are not our primary objectives.

Now, Issues of power consumption and processing load are not limited to resource-constrained devices. These issues also apply to entities constituting a wide area network where various local networks for different types of devices are inter-connected. The above-mentioned aggregation technique in section 2.3 is, however, only applicable for a specific sensor network. A fixed type 
of data, such as temperature or power usage, is assumed so that it can add or take the average for those data in this example. If we allow various types of data, devices, and interconnects of a large number of such devices, the above technique incurs difficulties. A wide area network, such as the Internet, has such characteristics and also must handle various communication patterns and routing paths. For example, the above technique only takes into account one-way aggregation. If we want to aggregate packets at some part of a wide area network but do not want to for some other parts, we cannot differentiate both cases if we use the above technique. Another problem is that for a local sensor network, we can assume a unique single destination. Sensors usually send data to a single sink in a local network. We then can assume that the final destination can perform some complicated processes on the aggregated data. This means we only need a simple operation for intermediate nodes. On the other hand, a wide area network has nodes with different capabilities. We cannot assume a single destination node for the Internet. Therefore, if we perform a specific operation in a node, it affects the overall network and negatively affects a large amount of data flowing through the Internet. Furthermore, in a local network, irreversible operation is an option. For example, if we only need the average value of an area, we can aggregate sensor data by taking their average. For a wide area network, we usually do not assume that a network changes the data content. Therefore, we cannot adopt such irreversible operation for a wide area network.

\section{Characteristics and Requirements for Wide Area Network}

In this section, we assume that quite a few short packets traverse through the wide area Internet. These short packets include local aggregated packets discussed in the previous section or the original short packets produced by sensor devices. We do not consider the individual results of aggregation done in local area networks and only consider the case in which there are an extremely large number of short packets flowing in a wide area network

The uni-directional aggregation technique discussed in the previous section works well for uniform and fixed local sensor networks. As we mentioned in the previous section, it does not function in a wide area network in which there are many destinations, routes, local networks, and a large amount of information (Figure 3). With this diversity in wide area networks, the following aggregation requirements need to be met.

\section{Multi-directionality}

To allow various combinations of devices and applications, we need to aggregate packets with different destinations and disaggregate them.

\section{Bi-directionality}

Bi-directional communication is required to support message transmissions both from IoT devices to a server and from a server to IoT devices. This makes it possible to enable remote management for sensors and feedback control for actuators.

\section{Selectivity}

Some applications require real-time transmission. Uniform aggregation will increase delay and negatively affect those applications. Therefore, we have to aggregate packets selectively by taking into considering individual types of applications. 


\section{Adaptation to node capability}

We allow nodes that cannot correctly process the aggregated packets. This means that some nodes do not have aggregation or disaggregation functions.

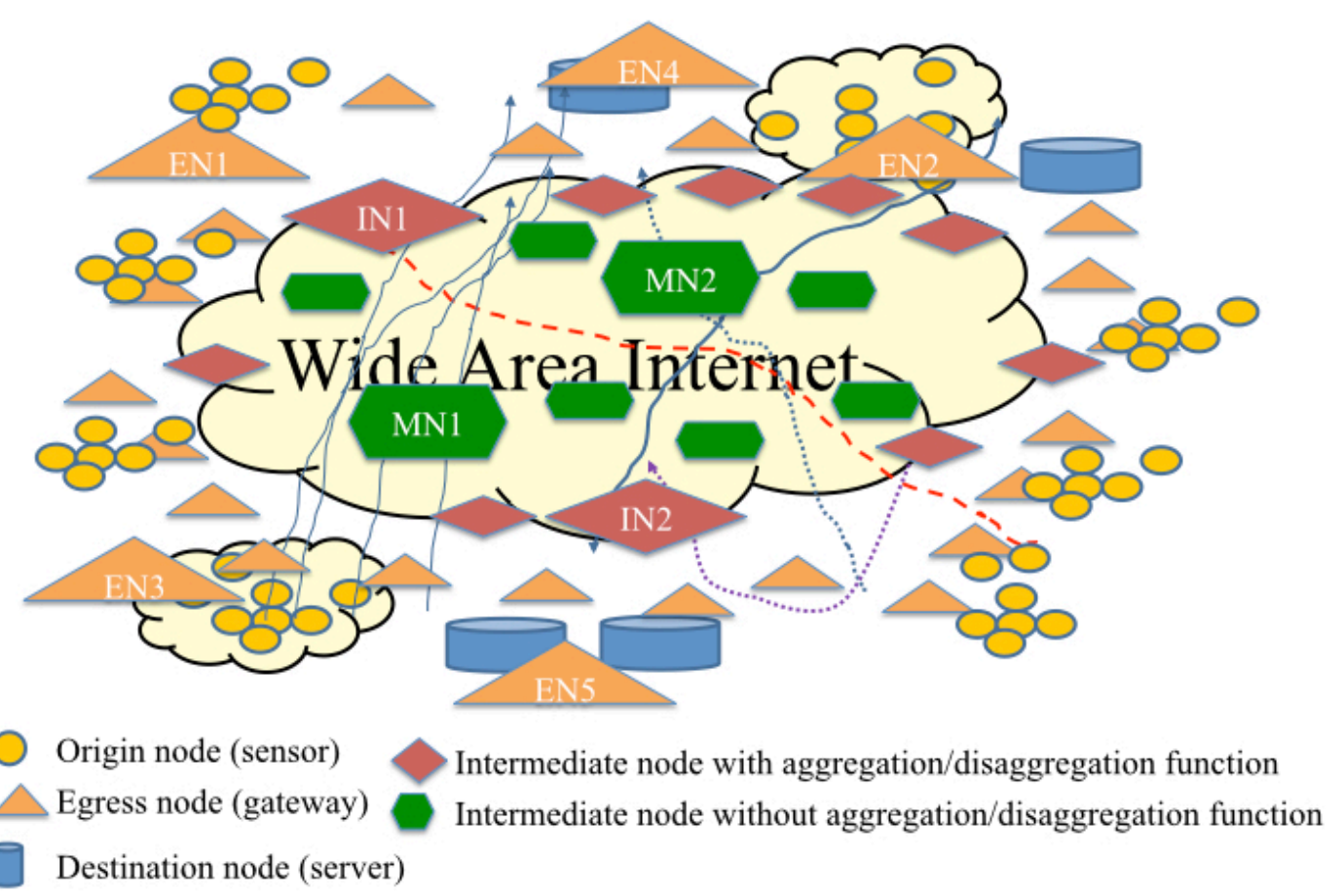

Figure 3: Wide area network supporting IoT

These requirements are different from those for a local network; thus a new scheme is needed that meets the above requirements.

\section{Aggregation Scheme for Wide Area Networks}

We describe our proposed aggregation scheme that meets the requirements mentioned in the previous section and its architecture.

\subsection{Design for aggregation scheme}

To meet requirements 1 and 2, intermediate nodes need to support both aggregation and disaggregation capabilities for packets with different destinations. Namely, when a node, such as a router, receives multiple packets, it aggregates them into a single packet (aggregation function). When it receives an aggregated packet, it disaggregates it and performs message-by-message processing (restoration function). 
We can meet requirement 3 by explicitly indicating allow or deny by using a flag in a packet. According to the flag, an intermediate node can aggregate packets if it is allowed. A node transmits a message that provides a way to prohibit aggregation along the path to the destination. This makes it possible to control the behavior of nodes depending on the characteristics of the message or requirements from the application that uses those data. This flag also prevents unnecessary aggregation.

To meet requirement 4, we need the following procedure. A node that must transfer aggregated packets checks if the next hop node has the capability to process the aggregated packets. If the next hop nodes support such capability, the initial node aggregates the packets and transfers them to the next node. A node declares its supporting capabilities of aggregation and disaggregation, then a source node can check if an intermediate node has restoration capability.

By implementing these four requirements, we can achieve non-unidirectional and complex aggregation and partial aggregation in a wide area network.

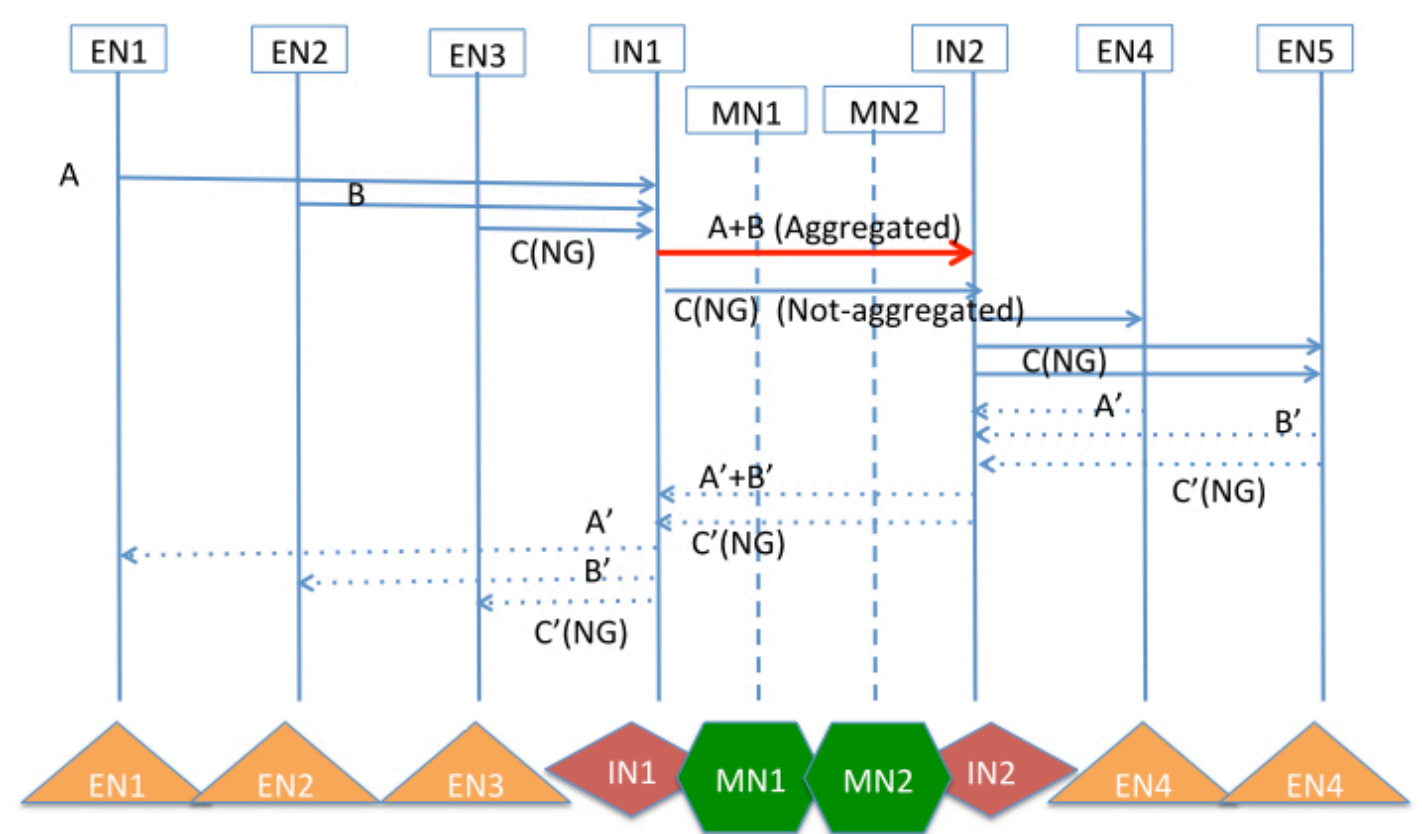

Figure 4: Example of packet aggregation flow

We illustrate our packet aggregation scheme in a wide area network with the above capabilities in Figure 4. The origin nodes (EN1, 2, and 3) send packets. Examples of origin nodes are the egress equipment of local area networks such as home gateways or IoT gateways. Intermediate nodes IN1 and 3, which correspond to the proxy in the application layer, aggregate packets received in a fixed time interval or disaggregate them. Intermediate nodes IN1 and 2 usually correspond to edge routers in the Internet. The IN1 node bundles and aggregates short packets with the same destination address or destination servers in the same autonomous system (AS) domain. There is another kind of intermediate node (MN1 and 2). These nodes do not support aggrega- 
tion or disaggregation capabilities. They only transfer packets to the next node. We assume these nodes are backbone routers in the wide area Internet. End nodes EN4 and 5 receive packets and are border routers with other Internet service providers or data centers. At this point, the node checks if the next node has the capability to process aggregated packets. It also excludes packets with a prohibit flag from the aggregation. It disassembles packets with different destinations and transfers them as separate packets. It performs the same process for the acknowledgement packets in the reverse direction. Note that in a local area sensor network, each node may change its role, such as an end or an intermediate node, depending on the network configuration. We assign a fixed role for each node for wide area networks.

With this architecture, we can apply an aggregation technique for the wide area Internet. Unlike the local area sensor network, we do not allow irreversible aggregation for a wide area network. We only aggregate packets and do not change the information in a packet. Therefore, our scheme is reversible and preserves the information in the payload.

Our aggregation scheme enables reduction of packet processing loads and power consumption as well as efficient use of bandwidth by avoiding an inflow of short packets for a wide area network. Note that we need processing power for aggregation and disaggregation. If we perform these processes on every node, the overall power consumption increases. We need to limit the number of nodes performing these processes. Therefore, as examples of these nodes with aggregation and disaggregation capabilities, we select gateway nodes at the border of different types of networks, such as IoT gateways located at the edge of a core network or border gateway to a data center. Then, the core nodes MN1 and 2 in Figure 4 only handle aggregated packets; thus, we can reduce the processing load for these nodes.

We show our proposed packet format using Internet Protocol (IP) and User Datagram Protocol (UDP)) for the transport layer protocol. In this case, we aggregate messages in CoAP layer (Figure 5).

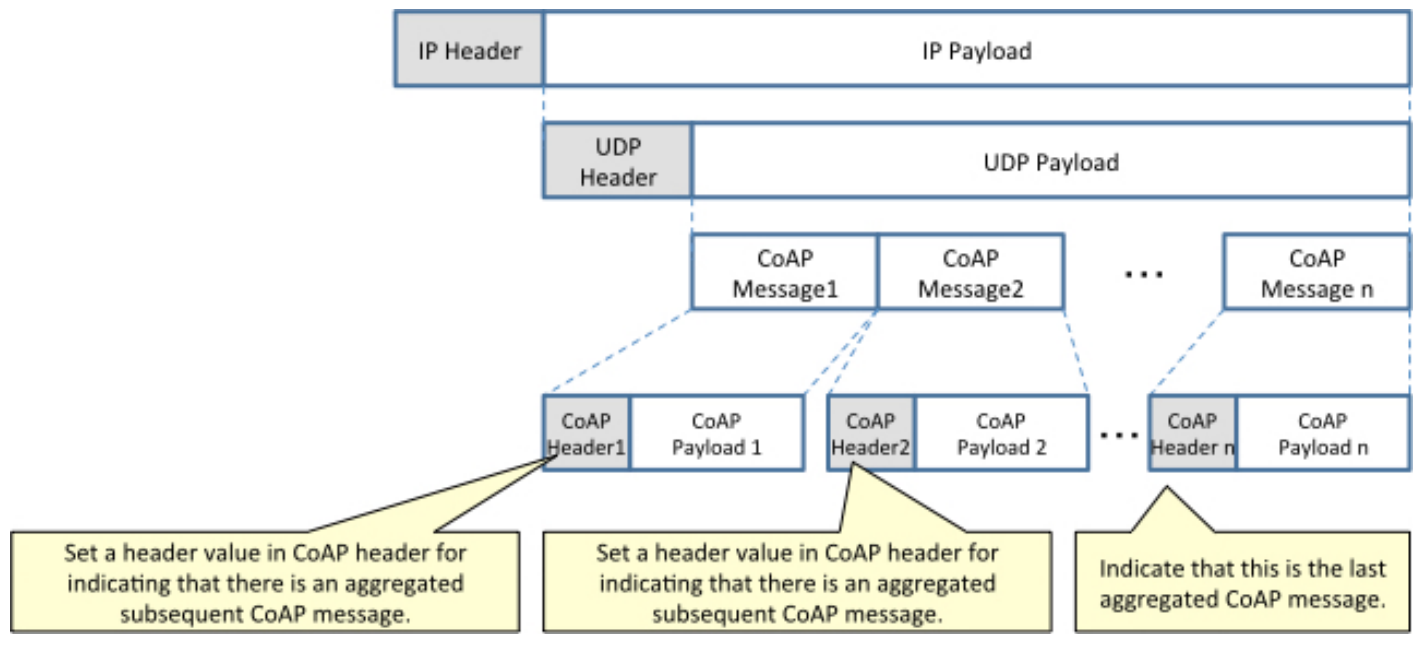

Figure 5: Example of packet format for aggregation 


\subsection{Aggregation and disaggregation procedures}

We explain our message aggregation process as follows. We first identify the target group of messages. More specifically, we determine among which receiving messages we aggregate or among which messages in queue we aggregate. This identification is based on destination IP addresses or a next-hop router addresses. Then, we start aggregation process. We concatenate multiple packets and create a single packet at the node. Finally, the node transmits the aggregated packet.

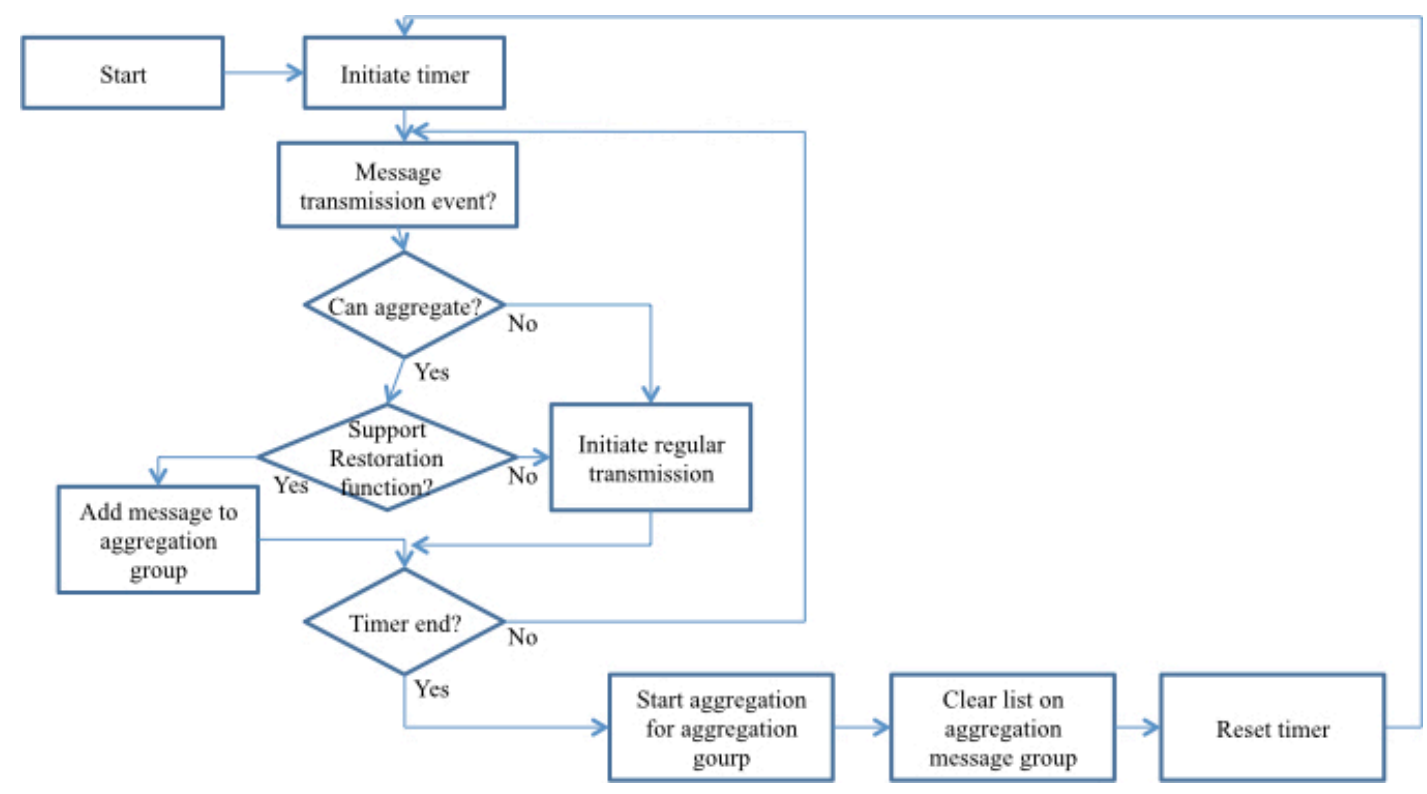

Figure 6: Flow for identifying messages for aggregation

We next explain the procedure for queuing the received packet for aggregation or disaggregation (Figure 6). When a node start running, it initiates a timer for aggregation. If the node supports restoration function, it disaggregates messages that it receives and puts the resorted message for the processing queue. The node repeats the above process until the aggregation timer expires. We here make it possible to multiple aggregations by putting once disaggregated packets to a processing queue for the sake of re-aggregation with other messages. When the timer for aggregation expires, we rest the timer and initiate a process for identifying messages for aggregation from the processing queue. Then restart the timer for receiving next messages. We repeat this process at the node.

We next explain the procedure for identifying aggregation messages from the processing queue (Figure 7). Right after the beginning of the process, the process picks up a message from the processing queue. It checks the destination of the message and if the destination is the node itself, it does not need to transfer the packet so the node invokes the regular process for receiving a message. If the message needs to transfer another node or a destination, we check if it has a flag for prohibit aggregation. If the message is allowed aggregation, the node checks whether the 
next-hop node support restoration function. If the next-hop node does not support restoration function, the source node invokes regular packet transfer process. If the next-hop node supports the restoration function, the source node adds the message to the target group of aggregation, which is prepared per destination. When there is no existing message for aggregation in the target aggregation group, the message becomes the first message for the target aggregation message group. The source node then checks if the processing queue is empty. When it is empty, it starts aggregation process per destination based target aggregation message group that has more than one message. If the process queue still has entry, it repeats the similar process by picking up a next message. When the size of the aggregated packets exceeds the allowed maximum size of a packet (Path Maximum Transfer Unite (MTU)) or predefined maximum size, it finishes aggregation process and transfers the packet.

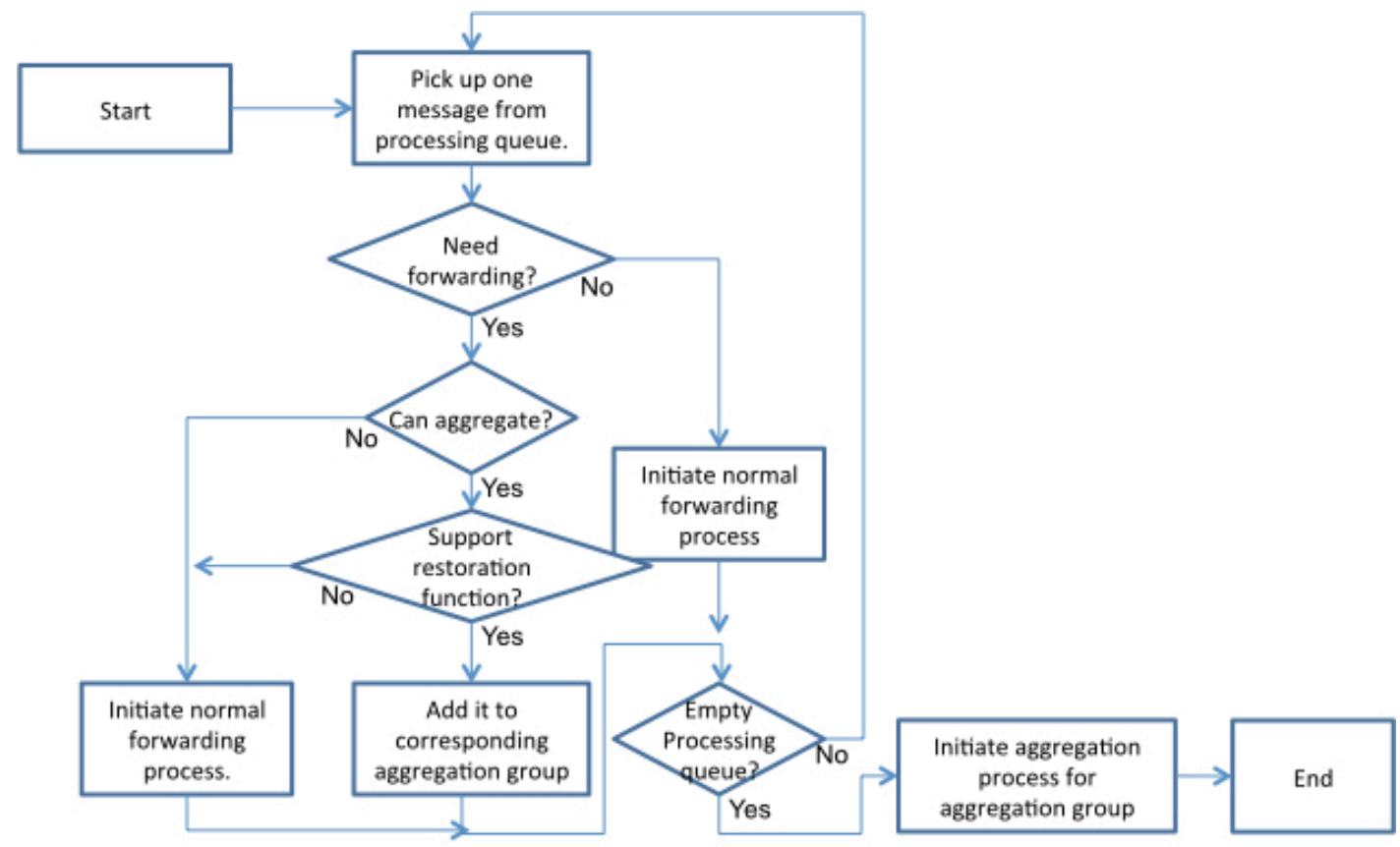

Figure 7: Identifying aggregation message

Message aggregation process is shown in Figure 8. It first determines aggregation order for $\mathrm{n}$ messages. After initializing a counter $k$, it read message $k$ and $k+1$ and calculate the aggregate packet size S. If it exceeds the maximum path MTU size, it transfers the aggregate packet at that time. If not, it continues this process by incrementing $\mathrm{k}$. 


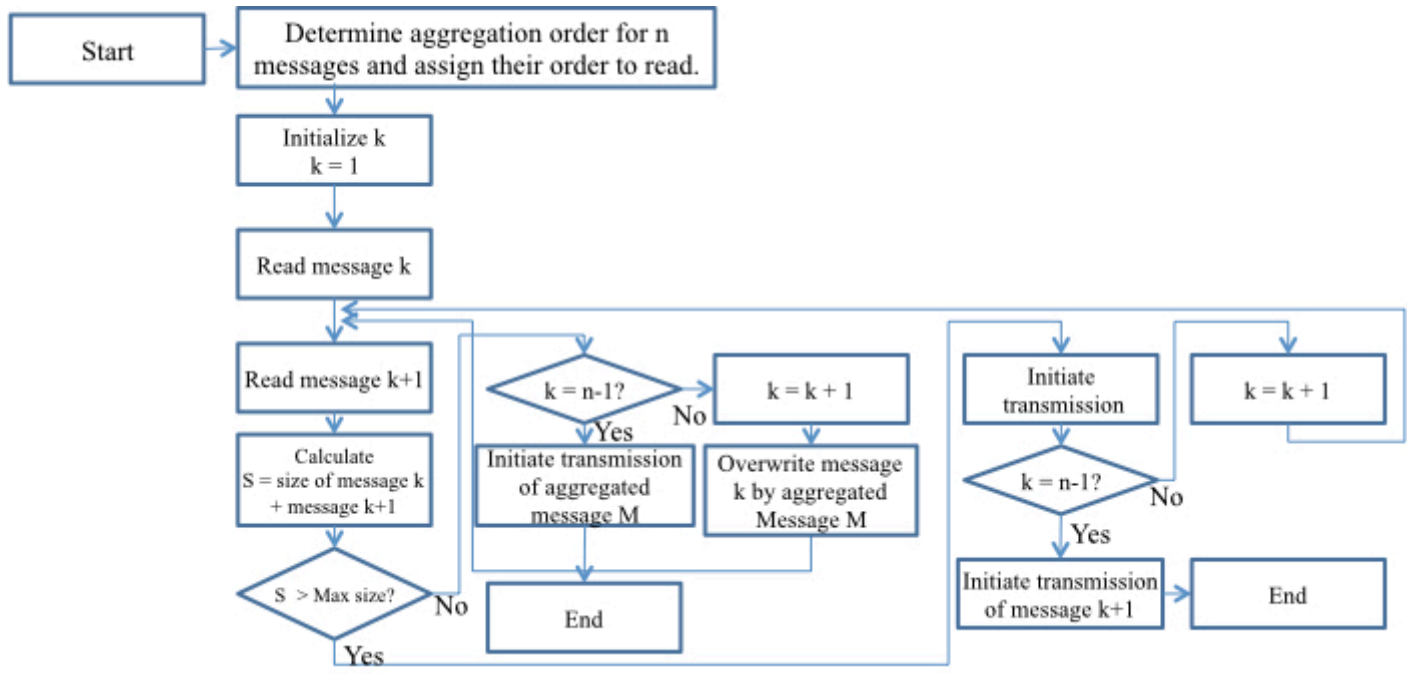

Figure 8: Message aggregation process

\subsection{Identifying router capabilities for aggregation and disaggregation}

To determine if a node can send an aggregated message to the next-hop or destination, the node needs to know if the next-hop node can restore the individual aggregated message. In other words, we have to identify the next-hop node that has the restoration function. There are many methods for achieving this. For this purpose, we can use the same communication protocol with the IoT message. We can also use other communication protocols. Or, we can adopt a Publish/Subscribe scheme for this [14]. In Figures 9 and 10, we show our CoAP-based implementation for inquiry, response, and notify messages for indicating the support of the restoration function. Note that we include text-based message for ease of understanding in these figures, but the CoAP is a binary encoded protocol so the actual CoAP payload does not show any text message. Figure 8 shows an inquiry message for the support of the disaggregation function. 


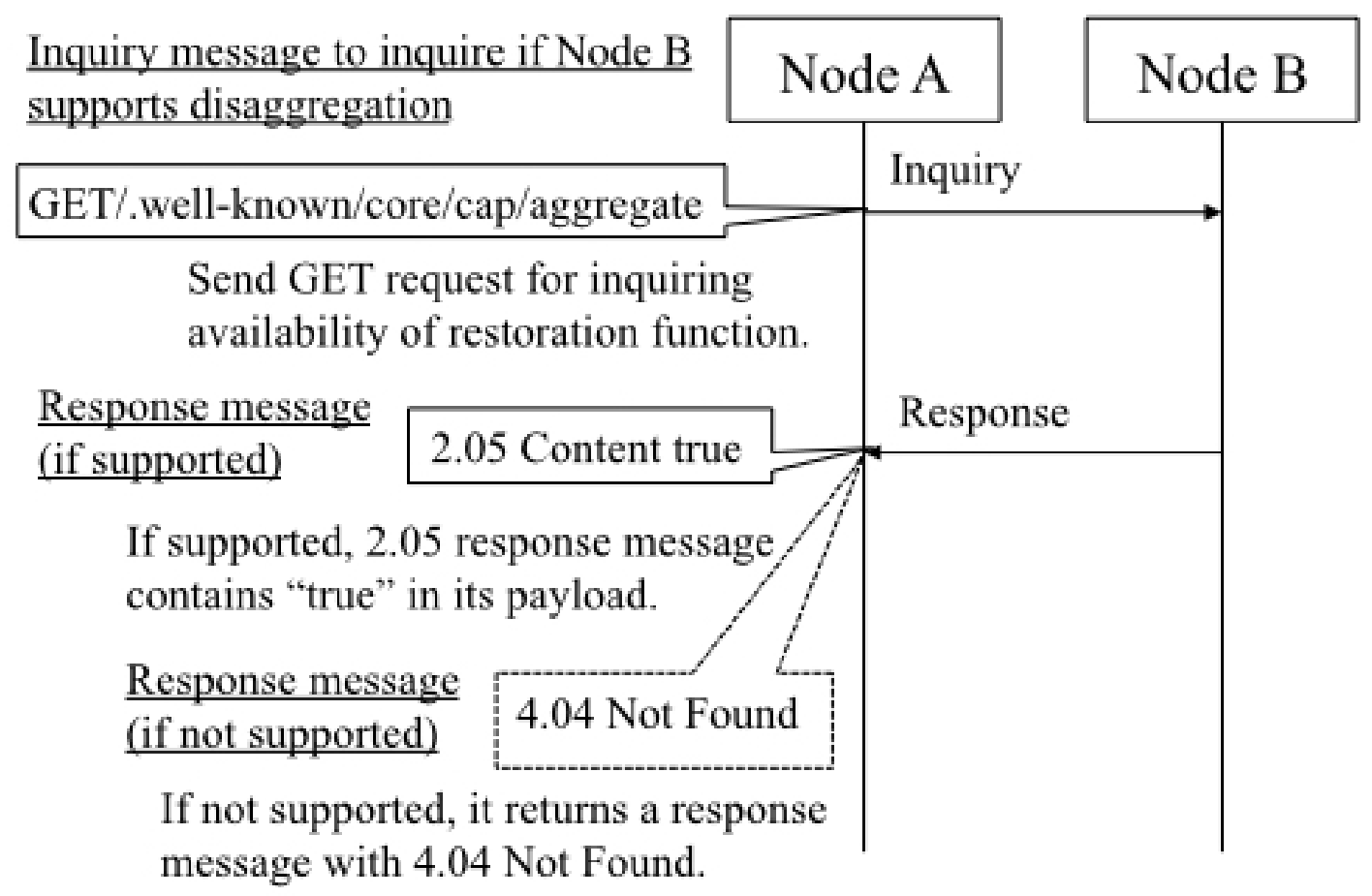

Figure 9: Inquiry and responses using CoAP

Notify message to tell the capability of Node B

\section{GET/test/test 1}

Aggregate: 3

Option 3 in this illustration means Node B supports disaggregation function. This message can be piggy backed.

Response message to confirm Notify

\subsection{Content value 1}

Figure 10: Notify using CoAP 
The source node sends this message to the adjacent or further ahead node if the recipient of this message supports the disaggregation function. This message exchange occurs independent of the user data transfer; it is sent periodically or at the time of route-information renewal. Using this message exchange, Node A can gather up-to-date information of the capabilities of its nearby nodes. Figure 9 shows a notify message. A node uses this message to advertise its supporting capabilities to adjacent nodes. If a node changes its supporting capability depending on its load, it can use this notification message.

Table 1: Description of CoAP aggregate option for message aggregation, restoration, prohibition of aggregation

Example of Aggregate option

\begin{tabular}{|c|c|c|c|c|}
\hline Option name & Option number & format & Length (byte) & default \\
\hline Aggregate & 2 & Unsigned int & 1 & 0 \\
\hline
\end{tabular}

Examples of Aggregate option number

\begin{tabular}{|c|l|}
\hline Option number & \multicolumn{1}{|c|}{ Meaning } \\
\hline 0 & $\begin{array}{l}\text { Non-aggregated single message or last message of aggregated } \\
\text { messages and no other subsequent message is connected. }\end{array}$ \\
\hline 1 & $\begin{array}{l}\text { Part of aggregated message and there are other connected subsequent } \\
\text { messages. }\end{array}$ \\
\hline 2 & Prohibit aggregation with other messages. \\
\hline 3 & A node that sent this message supports restoration function. \\
\hline
\end{tabular}

We can use either the in-band or out-of-band method for this information exchange. Table 1 shows a case in which the in-band method relays on the CoAP. We define the aggregate option as a CoAP option. With a value specified by this option, we represent the supporting capability. If we uniquely define the value of this option within a network management domain, we can use the CoAP without converting it to another protocol in a wide area network. Since we use the CoAP for carrying IoT data traffic, CoAP-based in-band message exchange means we can piggyback messages such as inquiry and notify on the data traffic.

\section{Evaluation on Power Consumption}

In this section, we evaluate how the aggregation scheme reduces the power consumption of the entire network. Our objective does not aim to reduce single router power consumption. As we can easily recognize, adding a new function at edge router increase the power consumption. However, our strategy is to cut the total power consumption for the entire network. In [15], they measure power consumptions for commercial routers. Their findings include that smaller the 
packet size is, the larger it consumes energy. Thus we can expect that if we use larger packet size in wide-area network, we might be able to compensate the energy increase incurred by aggre-gation function at edge routers. In order to validate this expectation, we rely on the model es-tablished in [16]. They model the power consumption of a router by that of measuring software router in detail. They analyze which part of the router consumes energy by what kind of relationships. They identified that the total energy consumption is calculated as the summation of (1) CPU usage, (2) Memory usage, (3) Interface usage, and (4) energy usage during idle status. They empirically derived several key parameters and create a total energy consumption model as a function of packet forwarding rate. They create their model for analyzing caching function but their model can be used our case as well. This is because although their scope is for evaluation of caching function in Information Centric Network, we can utilize their model for our packet aggregation function as both of them need to process packets above layer 4. By comparing their model, we adopt almost all values of parameters in [16] except calculations for clock cycles of aggregation and forwarding rate. We use 7120 for their summation of $F_{1}, 0$ for $F_{2}$, and 9612 for their summation of $F_{3}$ in their equation (5).

We assume a simple 4-node model for our evaluation; two edge routers and two core routers. For core routers without aggregation function, we assign 2039 for $F_{3}$ and set all 0 for $F_{1}$ and $F_{2}$ to model that it does not perform aggregation function. We assume 4 CPU cores for our routers.

We assume the size of IoT short packet as 46 byte and aggregated frame size is 1500 bytes. Therefore, about 30 short packets can be aggregated into a packet. By this assumption, we vary the input rate of the packet from $100 \mathrm{Mbit} / \mathrm{s}$ to $10 \mathrm{Gbit} / \mathrm{s}$. Note that we use the software router for both our edge and core routers as we anticipated massive deployment of Network Function Virtualization (NFV) and software routers will widely be used in core network as well. The result is shown in Figure 11.

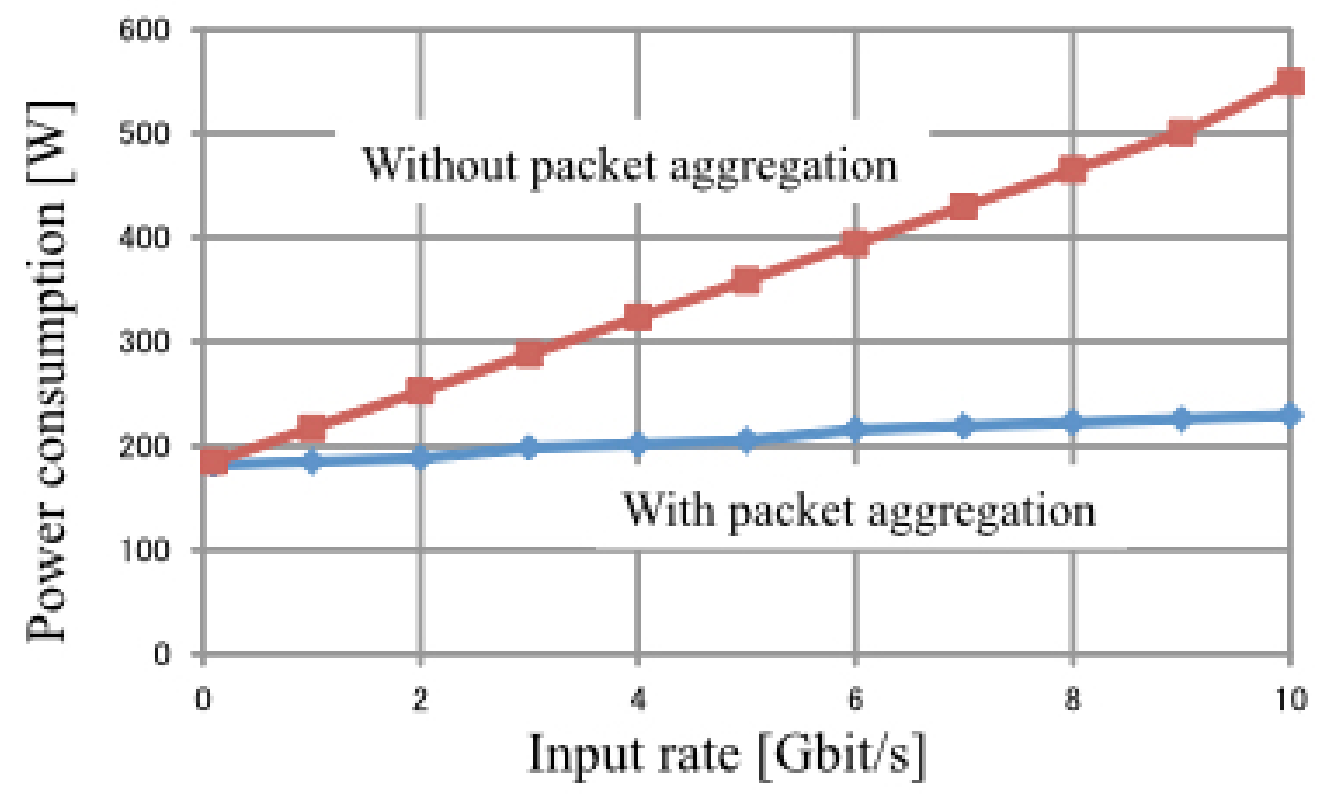

Figure 11: Comparison of power consumption with and without aggregation

As we can see from this figure, for small input rate, the difference for out reduction is small but 
the more increase the input rate, the more difference we observe in power consumptions. Although our evaluation is only one simple limited case and actual network configuration is more complex, we can see how packet aggregation can save the energy of the entire network.

By observing this result, we also are able to point out that workload reduction within wide-area network is closely related to power consumption of the entire network.

\section{Related works}

There are numerous works considering packet aggregation techniques. Even by limiting to their area on IoT networkings, many of them are focusing on wireless sensor networks. The authors of [11] discuss gateway placement problems for wires adhoc networks to optimize delay performance. In [12], they discuss data aggregation using anycast technique and design a protocol without maintaining explicit network structure. [17] discusses performance improvement by packet aggregation technique between a single segment of IEEE 802ac. Those works are focusing on performance improvement for wireless networks from various aspects but not considering end-to-end IoT networking.

In [18], they try to formulate packet aggregation technique using queueing model and consider how to minimize the system utilization. They modeled single entry network and mainly consider delay performance and system utilization.

The most relating work with us is [19]. They focused on end-to-end network and especially targeting IoT applications with small size packet to improve the performance of wide-area networks. Their objective is, however, to minimize the end-to-end delay of each packet to support real-time IoT applications. We agree with them that there exist some such use cases for IoT applications but in many cases, we believe IoT applications creating small packets are non-real-time one.

Our contribution of this paper is that we formulate a network architecture supporting end-to-end IoT networks filled with small packets to a wide-area network. Since small packet is harmful for router performance, our objective is to mitigate the degradation of network performance and thus energy consumption of the wide-area network. We then realize this architecture using CoAP-based implementation and showed the algorithm for aggregation and disaggregation. We also evaluate how packet aggregation reduces the power consumption in wide-area network.

\section{Conclusion}

We proposed a packet aggregation scheme and described the requirements and an implementation for applying it to a wide area network. With our proposed aggregation scheme, we can reduce the burden on the routers in core network in the wide area Internet due to the huge amount of short packets.

From the architectural viewpoint, our scheme creates overlay networks. For small packets aggregated into a larger packet, they cannot perceive the existence of intermediate core routers. Therefore, there are overlay networks on the wide area Internet. Aggregation and Disaggregation 
points are the nodes for these overlay networks. We anticipate the emergence of a huge number of IoT devices connecting to the Internet. By constructing overlay networks, we not only can reduce the packet processing load in a router but also can create a logical network over the Internet. We can define this logical network based on the types of information created by devices. Different types of information have different requirements for network performance, such as delay and loss. If we can aggregate appropriately, we can create multiple logical networks with different characteristics. If we further extend the logical network to implement the meaning of sensor information, we can achieve networking based on the meaning or value of information [20].

We implemented our proposed scheme by extending CoAP as a type of transport layer. This approach treats CoAP as the end-to-end convergence layer. Therefore, our approach maintains end-to-end transparency at the transport layer level. It will be an appropriate solution for service providers who want to directly control their remote IoT devices. When we extend this approach for more general information centric networking with multiple service providers sharing the same IoT devices, consideration on the appropriate layers is left for a further study item from the viewpoint of logical networking by using aggregation.

One might think adding many rich capability overloading edge routers. We, however, thinks that recent edge routers can enhance their capability by adding daughter boards with CPUs or NPUs and thus additional functions can be supported by those daughter board without degrading their forwarding capability. In near future, we further anticipate massive deployments of Network Function Virtualization (NFV) technology for router. Therefore, many routers will be virtualized and located in datacenters supported by scalable server technologies. Of course this increase the power consumption at edge routers but on the other hand, as we showed in section 5 , we can reduce the entire network energy consumption.

\section{References}

[1] R. Fielding, J. Gettys, J. Mogul, H. Frystyk, L. Masinter, P. Leach, and T. Berners-Lee, "Hypertext Transfer Protocol -- HTTP/1.1," RFC2616, IETF, 1999.

[2] Z. Shelby, K. Hartke, and C. Bormann, "The Constrained Application Protocol (CoAP)," RFC7252, IETF, 2014.

[3] R. Ishibashi, T. Ohba, and A. Koike, "A packet aggregation method in wide area M2M/IoT networks," Proc. IEICE General Conference, B-7-54, 2013, pp. 198.

[4] H. Schulzrinne, S. Casner, R. Frederick, and V. Jacobson, “RTP: A Transport Protocol for Real-Time Applications," IETF RFC3550, 2003.

[5] ITU-T, "B-ISDN ATM Adaptation Layer specification: Type 2 AAL," Recommendation I.363.2, September 1997.

[6] ITU-T, "Segmentation and Reassembly Service Specific Convergence Sublayer for the AAL type 2," Recommendation I.366.1, June 1998. 
[7] ITU-T, "Pulse code modulation (PCM) of voice frequencies," Recommendation G.711, November 1988.

[8] IEEE, "Local and metropolitan area networks-- Specific requirements-- Part 11: Wireless LAN Medium Access Control (MAC) and Physical Layer (PHY) Specifications Amendment 5: Enhancements for Higher Throughput, ” 802.11n, IEEE Standard for Information technology, 2009.

[9] J. Hui (Editor), and P. Thubert, "Compression Format for IPv6 Datagrams over IEEE 802.15.4-Based Networks,” RFC6282, IETF, 2011.

[10] IEEE Computer Society, “IEEE Std. 802.15, 4-2006,” 2006.

[11] J. L. Wong, R. Jafari, and M. Potkonjak, "Gateway placement for latency and energy efficient data aggregation," 29th Annual IEEE International Conference on Local Computer Networks, 2004, pp. 490- 497.

[12] K.W. Fan, S. Liu, and P. Sinha, "Structure-Free Data Aggregation in Sensor Networks," IEEE Transactions on Mobile Computing, Vol. 6, Issue 8, 2007, pp. 929 - 942.

[13] E. Mlaih, and S. A. Aly, "Secure Hop-by-Hop Aggregation of End-to-End Concealed Data in Wireless Sensor Network,” Proc. 2nd IEEE MCN'08 workshop, Phoenix, AZ, 2008.

[14] P.T. Eugster, P.A. Felber, R. Guerraoui, and A.M. Kermarrec, "The many faces of publish/subscribe,” ACM Computer Survey, vol.35, no.2, 2003, pp.114-131.

[15] J. Chabarec, J. Sommers, P. Bardord, C. Estan, D. Tsiang, and S. Wright, "Power Awareness in Network Design and Routing” Proc. of The 27th Conf. on Computer Communications, 2008, DOI: 10.1109/INFOCOM.2008.93.

[16] T. Hasegawa, Y. Nakai, K. Ohsugi, J. Takemasa, K. Koizumi, and I. Pasaras, "Empirically Modeling How a Multicore Software ICN Router and an ICN Network Consume Power," Proc. of the 1st ACM Conf. on Information-Centric Networking, 2014, pp. 157-166.

[17] B. Bellalta, J. Barcelo, D. Staehle, A. Vinel, and M. Oliver, "On Performance of Packet Aggregation in IEEE 802.11ac MU-MIMO WLANs," IEEE Communications Letters, Vol. 16, Issue 10, 2012, pp. 1588-1591.

[18] J.H. Hong, and K. Sohraby, "On Modeling, Analysis, and Optimization of Packet Aggregation Systems,” IEEE Trans. on Communications, Vol. 58, No. 2, 2010, pp.660-668.

[19] A. Sawabe, K. Tsukamoto, and Y. Oie, "QoS-aware Packet Chunking Schemes for M2M Cloud Services” 28th Int'l Conf. on Advanced Information Networking and Applications Workshops, 2014, pp. 166-173.

[20] V. Jacobson, D. K. Smetters, J. D. Thornton, M. F. Plass, N. H. Briggs, and R. L. Braynard, "Networking named content," Proc. of the 5th Int'l Conf. on Emerging networking experiments and technologies, 2009. 